\title{
A simple technique for anchoring chest tubes
}

\author{
M.A. Rashid, T. Wikström, P. Örtenwall
}

\section{A simple technique for anchoring chest tubes. M.A. Rashid, T. Wikström, P. Örtenwall. CERS Journals Ltd 1998.}

ABSTRACT: Although some techniques have been described for securing chest tubes, many complications are still reported. A simple method is described for securing chest tubes and closure of the wound, resulting in good fixation of the tube and an airtight closure of the chest wall with a cosmetically satisfactory result. Eur Respir J 1998; 12: 958-959.
Division of Vascular and Trauma Surgery, Dept of Surgery, Sahlgrenska University Hospital/Östra, Gothenburg University, Gothenburg, Sweden.

Correspondence: M.A. Rashid, Trebackegatan 11, 41674 Gothenburg, Sweden

Keywords: Anchoring, chest tubes, spontaneous withdrawal

Received: November 101997

Accepted after revision March 231998
Chest tube thoracostomy is a common and very useful therapeutic procedure. It is indicated in pneumothorax with or without tension, traumatic haemothorax, haemopneumothorax, etc. However, it is not without risk, especially when aggressively used in trauma patients [1]. Chest tubes are inserted frequently by junior and relatively inexperienced clinicians from various specialities. The usual complications are malposition and obstruction, either by occlusion or by kinking. Ectopic insertion has been reported in all thoracic and upper abdominal organs. Tension pneumothorax is a rare complication due to clamping of the tube during active drainage, particularly with significant air leak or due to incorrect application of the Heimlich apparatus [2]. Recently, we have seen four cases with spontaneous dislodgement of chest tubes in our department.

Different methods are described for the security of chest tubes and closure of thoracostomy incisions [3-5]. A surgical tape can be used to cover the incision and anchor the tube close to the chest wall and an omental tag of tape can hold the tube close to the chest wall, allowing some motion of the tube without kinking. Additionally, the accessory tubing can be pinned to the bed for more security. In spite of all such precautions, spontaneous removal of chest tubes has been noticed in our institution. Therefore, an effective and simple method of anchoring the tube to the chest wall is warranted.

\section{Technique}

One author (M.A. Rashid) has used a technique of anchoring the tube for many years and never seen such a complication. This technique is simple. The third to the fifth intercostal space in the midaxillary line, just below the pectoralis major muscle in the axilla, is usually the site selected to avoid an apparent surgical scar. Under sterile conditions and after liberal infiltration of the periosteum of the adjacent rib and parietal pleura (1\% xylocaine, 10 $20 \mathrm{~mL}$ ), a $2 \mathrm{~cm}$ incision is made over and parallel to the midportion of the rib.
Two sutures (size 0 or 2-0) are placed at either side of the wound and a horizontal mattress suture is placed in the centre of the wound and knotted in the middle of its length (fig. 1). The sutures are placed at about $0.5 \mathrm{~cm}$ from the wound edges. The chest tube is inserted after blunt dissection and digital access to the pleural cavity to lyse any adhesions and to assure entrance of the pleural cavity. The tube is directed anteroapically for pneumothorax or posterobasally for fluid drainage. The sutures at either side of the wound are simply tied to close the wound and the free edges are tied around the tube (just above its entrance into the chest) as bilateral anchoring devices (fig. 2A and B). The mattress suture is wrapped several times around the tube (just above the other two sutures), then the free edges

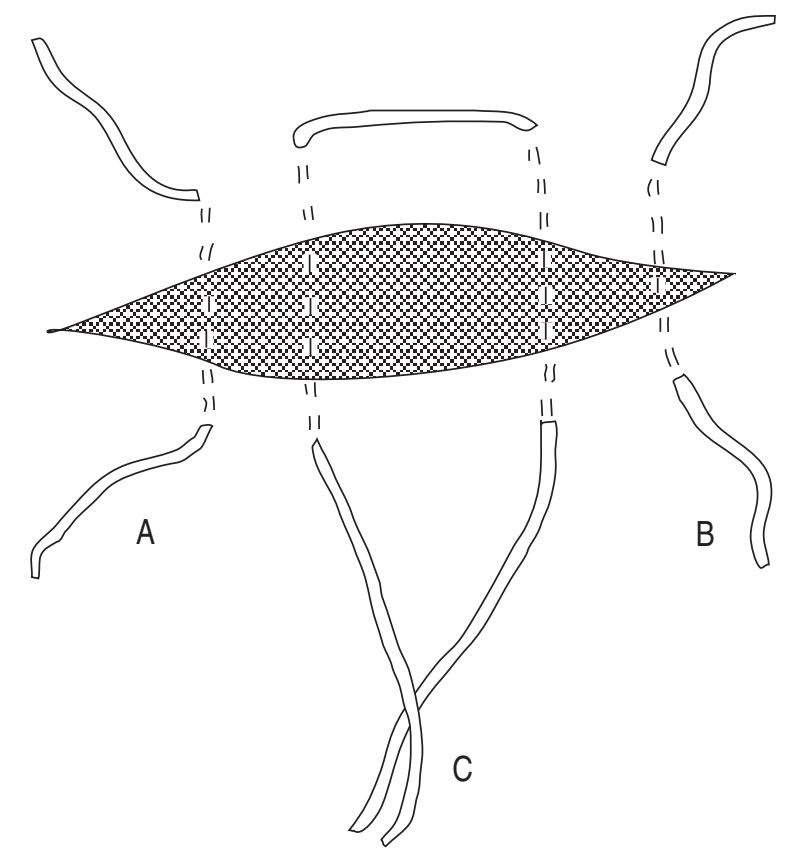

Fig. 1. - Preparation of the incision: sutures A and B at either side and a horizontal mattress stitch $\mathrm{C}$ in the middle of the wound. 


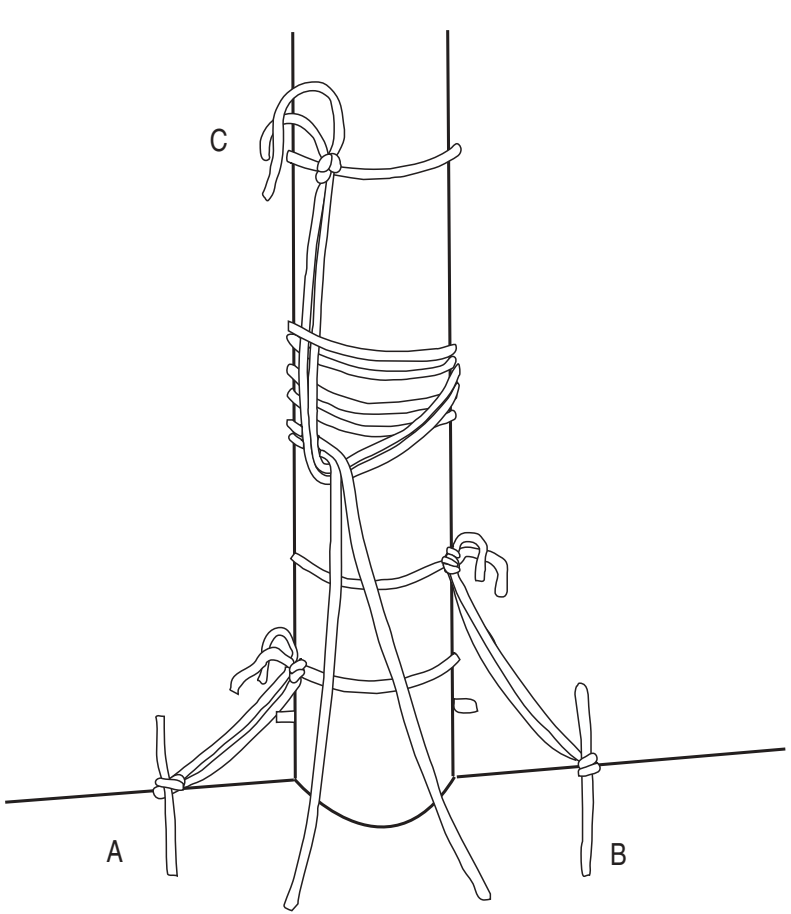

Fig. 2. - Tube insertion: sutures A and B are tied to close the wound and the free edges are secured around the tube. Stitch $\mathrm{C}$ is wrapped several times around the tube, then the free edges are grasped by a mosquito clamp from underneath the mattress suture and turned up to be secured around the tube.

are grasped by a mosquito clamp from underneath the mattress suture and turned up to be tied around the tube to anchor it (fig. 2C). A surgical tape is applied to cover the wound and secure the tube.

At the time of tube removal, the adhesive tape is withdrawn and the two side sutures are cut just above their knots and left closing the wound on either side (fig. 3A

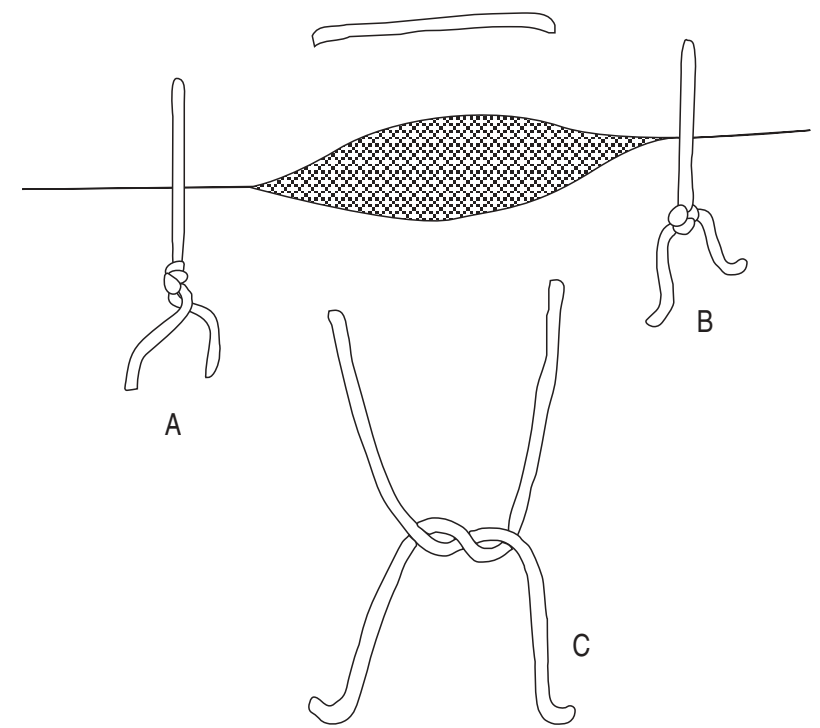

Fig. 3. - Tube removal: sutures A and B are cut just above their knots and left closing the wound on either side, suture $\mathrm{C}$ is cut below the knot and tension is applied on the free suture ends.

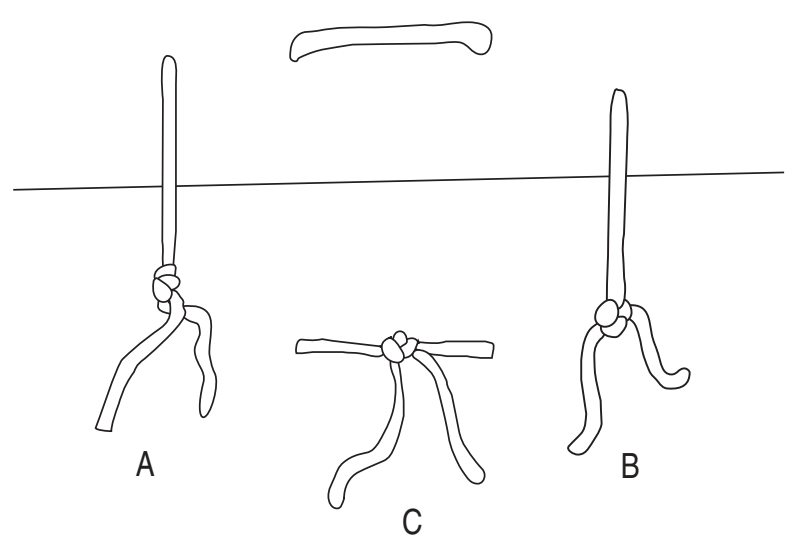

Fig. 4. - Final result: stitch $\mathrm{C}$ is tied to close the wound in a cosmetically satisfactory fashion.

and B). The mattress stitch is cut below the knot and tension is applied on the free suture ends to close the incision and prevent air entry (fig. 3C). While the patients hold their breath at the end of a deep inspiration and performs a Valsalva manoeuvre, the tube is removed quickly and smoothly. This suture is then tied to close the wound after tube removal with perfect approximation of the wound edges in a cosmetically satisfactory fashion (fig. 4).

\section{Discussion}

We believe that this technique is worth considering to ensure fixation of the chest tube to the thoracic wall and to prevent the dangerous consequences of chest tube separation. The three applied sutures secure the tube in position and prevent forwards and backwards movements into the pleural cavity. Delayed closure of the wound results in a cosmetically good scar, without the need for a new stitch, which is sometimes required upon chest tube removal. However, the wound should not be closed tightly in case of superficial infection of the chest tube site after removal, which is rarely seen. This technique is simple and easy to perform and gives safe fixation of the tube to the chest wall with a satisfactory result.

\section{References}

1. Millikan JS, Moore EE, Steiner E, Aragon GE, Van Way CW. Complications of tube thoracostomy for acute trauma. Am J Surg 1980; 140: 738-741.

2. Mainini SE, Johnson FE. Tension pneumothorax complicating small-caliber chest tube insertion: case reports. Chest 1990; 97: 759-760.

3. Simon RR, Bailey TD, Abraham E, Brenner B. A new technique for securing a chest tube. Ann Emerg Med 1982; 11: 619-621.

4. Roe BB. Improved technique for closure of thoracostomy incision. Surg Gyneol Obstet 1965; 121: 845-846.

5. Fledge JB. A simple technique for closing thoracostomy incisions. Surg Gynecol Obstet 1967; 124: 847-848. 